\title{
Finite element model for vibration analysis of pre-twisted Timoshenko beam
}

\author{
B. Yardimoglu*, T. Yildirim \\ Department of Mechanical Engineering, Izmir Institute of Technology, 35430 Urla, Izmir, Turkey \\ Received 1 May 2002; accepted 7 May 2003
}

\begin{abstract}
A new linearly pre-twisted Timoshenko beam finite element, which has two nodes and four-degrees-offreedom per node, is developed and subsequently used for coupled bending-bending vibration analysis of pre-twisted beams with uniform rectangular cross-section. First, displacement functions based on two coupled displacement fields (the polynomial coefficients are coupled through consideration of the differential equations of equilibrium) are derived for pre-twisted beams whose flexural displacements are coupled in two planes. This approach helps to reduce the number of nodal variables. Next, the stiffness and mass matrices of the finite element model are obtained by using the energy expressions. Finally, the natural frequencies of pre-twisted Timoshenko beams are obtained and compared with previously published theoretical and experimental results to confirm the accuracy and efficiency of the present model. Excellent agreement is found with the previous studies. Also, the new pre-twisted Timoshenko beam element has good convergence characteristics.
\end{abstract}

(C) 2003 Elsevier Ltd. All rights reserved.

\section{Introduction}

Pre-twisted beams are structural elements, used in many different systems, such as turbine blades, helicopter rotor blades, aircraft propeller blades, wind turbine blades. Many researchers analyzed twisted beams using different techniques. A carefully selected sample of the relevant literature is as follows: Exact solutions of Timoshenko's equation for simple supported uniform beams were given by Anderson [1]. The general equations of motion of a pre-twisted cantilever blade were derived by Carnegie [2]. Then Carnegie [3] extended his study for the general equations of motion of a pre-twisted cantilever blade allowing for torsion, bending, rotary inertia and

\footnotetext{
*Corresponding author. Tel.: + 90-232-498-6599; fax: +90-232-498-6505.

E-mail address: bulentyardimoglu@iyte.edu.tr (B. Yardimoglu).
} 
deflections due to shear. Dawson et al. [4] found the natural frequencies of pre-twisted cantilever beams of uniform rectangular cross-section allowing for shear deformation and rotary inertia by the numerical integration of a set of first order simultaneous differential equations. They also made some experiments in order to obtain the natural frequencies for beams of various breadth to depth ratios and lengths ranging from 3 to 20 in $(7.62$ to $50.8 \mathrm{~cm})$ and pre-twist angle in the range $0-90^{\circ}$. Carnegie and Thomas [5] investigated the effects of shear deformation and rotary inertia on the frequencies of flexural vibration of pre-twisted uniform and tapered cantilever beams by using the finite-difference method. Gupta and Rao [6] used the finite element method to determine the natural frequencies of uniformly pre-twisted tapered cantilever beams. Subrahmanyam et al. [7] applied the Reissner method and the total potential energy approach to calculate the natural frequencies and mode shapes of pre-twisted cantilever blading including shear deformation and rotary inertia. Rosen [8] presented a survey paper as an extensive bibliography on the structural and dynamic aspects of pre-twisted beams. Chen and Keer [9] studied the transverse vibration problems of a rotating twisted Timoshenko beam under axial loading and spinning about its axial axis, and investigated the effects of the twist angle, rotational speed, and axial force on natural frequencies by the finite element method. Chen and Ho [10] introduced the differential transform to solve the free vibration problems of a rotating twisted Timoshenko beam under axial loading. Lin et al. [11] derived the coupled governing differential equations and the general elastic boundary conditions for the coupled bending-bending forced vibration of a non-uniform pretwisted Timoshenko beam by Hamilton's principle. They used a modified transfer matrix method to study the dynamic behaviour of a Timoshenko beam with arbitrary pre-twist. Banerjee [12] developed a dynamic stiffness matrix and used it for free vibration analysis of a twisted beam. Rao and Gupta [13] derived the stiffness and mass matrices of a rotating twisted and tapered Timoshenko beam element, and calculated the first four natural frequencies and mode shapes in bending-bending mode for cantilever beams. Narayanaswami and Adelman [14] showed that a straightforward energy minimization yields the correct stiffness matrix in displacement formulations when transverse shear effects are included. They also stated that in any finite element displacement formulation where transverse shear deformations are to be included, it is essential that the rotation of the normal (and not the derivative of transverse displacement) be retained as a nodal degree of freedom. Dawe [15] presented a Timoshenko beam finite element that has three nodes and two-degrees-of-freedom per node, which are the lateral deflection and the cross-sectional rotation. The element properties were based on a coupled displacement field; the lateral deflection was interpolated as a quintic polynomial function and the cross-sectional rotation was linked to the deflection by specifying satisfaction of the moment equilibrium equation within the element. The effect of rotary inertia was included in "lumped" form at the nodes. He expressed that his model is superior to the basic and previous higher order models on a total degrees of freedom basis. Interested readers are referred to a book by Petyt [16], which gives the same technique based on coupled displacement field and the finite element applications to structural vibration analysis in great detail.

However, as far as the authors are aware, no work has been reported in the existing literature on finite element formulation of pre-twisted Timoshenko beam based on coupled displacement fields that facilitate modelling with a few nodal variables. On the other hand, it should be pointed out that the models based on independent displacement functions are required to include some derivatives of the nodal displacements, such as $\partial u / \partial z$ or $\partial v / \partial z$, as nodal variables depending on 
the number of coefficients employed in the displacement functions in order to determine the coefficients of these functions. Therefore, in the present study, a new finite element model as an extension of Dawe's study to pre-twisted Timoshenko beam is derived. The results are compared with those in the literature, and excellent agreement is observed.

\section{Stiffness and mass matrices of the finite element}

A uniformly pre-twisted beam having constant rectangular cross-section and the co-ordinate system used are shown in Fig. 1. Centroid of the cross-section of the undeformed pre-twisted beam lies on the $z$-axis. The angle between the axes $x$ and $x^{\prime}$ is $\phi$ (see Appendix $\mathrm{C}$ for a list of notation). Differential equations of the motion of a homogeneous isotropic pre-twisted Timoshenko beam for elastically coupled bending-bending vibrations are given in Appendix A, neglecting the warping of the cross-section. The dynamic displacements can be obtained by solving the equations of motion; however, since solutions depend on the previous time history of the nodal displacements, there are no simple relationships between dynamic displacements within the element and the nodal displacements and/or variables [16,17]. Therefore, the reasonable dynamic displacements are obtained by using the static displacements, while carrying all the timedependent terms in the formulations [16,18]. The finite element model derived here is based on explicit satisfaction of the homogeneous form of Eqs. (A.1)-(A.4). It is evident that the use of static displacements to obtain the approximate solution for dynamic condition calls for an increase in the number of elements required for a given accuracy of the solution. However, this is amply compensated by the simplicity of the mathematical analysis it provides [16]. Hence, in Eqs. (A.1)-(A.4), eliminating three parameters from the set $\left\{u, v, \theta_{x}, \theta_{y}\right\}$ in turn gives,

$$
\frac{\mathrm{d}^{4} u}{\mathrm{~d} z^{4}}=0, \quad \frac{\mathrm{d}^{4} v}{\mathrm{~d} z^{4}}=0, \quad \frac{\mathrm{d}^{3} \theta_{x}}{\mathrm{~d} z^{3}}=0, \quad \frac{\mathrm{d}^{3} \theta_{y}}{\mathrm{~d} z^{3}}=0 .
$$

Eq. (1) results in an element with constant shear forces along its length, linear variation of moments, quadratic variation of cross-sectional rotations and cubic variation of transverse displacements. Therefore, the general solutions of these four equations are chosen as polynomials in $z$, considering the time-dependent coefficients as follows:

$$
u(z, t)=a_{0}(t)+a_{1}(t) z+a_{2}(t) z^{2}+a_{3}(t) z^{3},
$$

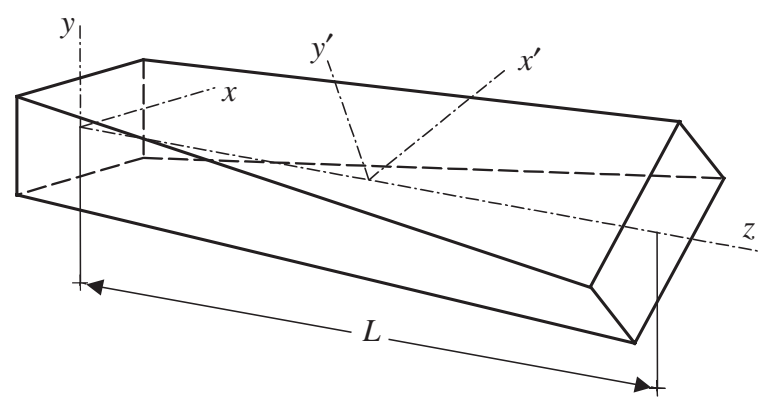

Fig. 1. Uniformly pre-twisted constant cross-sectional beam. 


$$
\begin{gathered}
v(z, t)=b_{0}(t)+b_{1}(t) z+b_{2}(t) z^{2}+b_{3}(t) z^{3}, \\
\theta_{x}(z, t)=c_{0}(t)+c_{1}(t) z+c_{2}(t) z^{2}, \\
\theta_{y}(z, t)=d_{0}(t)+d_{1}(t) z+d_{2}(t) z^{2} .
\end{gathered}
$$

Eqs. (2)-(5) represent the spatial approximation of displacements for any time $t$. For simplicity, $(z, t)$ and $(t)$ will be omitted from the formulations throughout the text. Using homogeneous form of Eqs. (A.1)-(A.4), the relationships are obtained linking $u, v, \theta_{x}$ and $\theta_{y}$ in the form

$$
\frac{\mathrm{d}}{\mathrm{d} z}\left(E I_{x x} \theta_{x}^{\prime}+E I_{x y} \theta_{y}^{\prime}\right)+k A G\left(v^{\prime}-\theta_{x}\right)=0, \quad \frac{\mathrm{d}}{\mathrm{d} z}\left(E I_{y y} \theta_{y}^{\prime}+E I_{x y} \theta_{x}^{\prime}\right)+k A G\left(u^{\prime}-\theta_{y}\right)=0 .
$$

The area moments of inertia of the cross-section should be noted as follows:

$$
\begin{aligned}
& I_{x x}(z)=I_{x^{\prime} x^{\prime}} \cos ^{2} \phi(z)+I_{y^{\prime} y^{\prime}} \sin ^{2} \phi(z), \\
& I_{y y}(z)=I_{y^{\prime} y^{\prime}} \cos ^{2} \phi(z)+I_{x^{\prime} x^{\prime}} \sin ^{2} \phi(z), \\
& I_{x y}(z)=0.5\left(I_{y^{\prime} y^{\prime}}-I_{x^{\prime} x^{\prime}}\right) \sin 2 \phi(z),
\end{aligned}
$$

where $\phi(z)=\phi_{0}+\theta z$.

By using Eqs. (2)-(6) the coefficients $c_{0}, c_{1}, c_{2}, d_{0}, d_{1}$ and $d_{2}$ can be expressed in terms of the coefficients $a_{0}, a_{1}, a_{2}, a_{3}, b_{0}, b_{1}, b_{2}$ and $b_{3}$ by equating coefficients of powers of $z$. This procedure yields:

$$
\begin{array}{lll}
c_{0}=\beta_{1} a_{2}+\beta_{2} a_{3}+b_{1}+\beta_{3} b_{2}+\beta_{4} b_{3}, & c_{1}=\beta_{5} a_{3}+2 b_{2}+\beta_{6} b_{3}, & c_{2}=3 b_{3}, \\
d_{0}=a_{1}+\beta_{7} a_{2}+\beta_{8} a_{3}+\beta_{1} b_{2}+\beta_{2} b_{3}, & d_{1}=2 a_{2}+\beta_{9} a_{3}+\beta_{5} b_{3}, & d_{2}=3 a_{3},
\end{array}
$$

where

$$
\begin{gathered}
\beta_{1}=\frac{2 E}{k A G} I_{x y}^{\prime}, \quad \beta_{2}=6\left(\frac{E}{k A G}\right)^{2} I_{x y}^{\prime}\left(I_{x x}^{\prime}+I_{y y}^{\prime}\right)+\left(\frac{6 E}{k A G}\right) I_{x y}, \\
\beta_{3}=\frac{2 E}{k A G} I_{x x}^{\prime}, \quad \beta_{4}=6\left(\frac{E}{k A G}\right)^{2}\left(I_{x x}^{\prime 2}+I_{x y}^{\prime 2}\right)+\left(\frac{6 E}{k A G}\right) I_{x x} \\
\beta_{5}=\frac{6 E}{k A G} I_{x y}^{\prime}, \quad \beta_{6}=\frac{6 E}{k A G} I_{x x}^{\prime}, \\
\beta_{7}=\frac{2 E}{k A G} I_{y y}^{\prime}, \quad \beta_{8}=6\left(\frac{E}{k A G}\right)^{2}\left(I_{y y}^{\prime 2}+I_{x y}^{\prime 2}\right)+\left(\frac{6 E}{k A G}\right) I_{y y} \\
\beta_{9}=\frac{6 E}{k A G} I_{y y}^{\prime}
\end{gathered}
$$

in which

$$
\begin{aligned}
& I_{x x}^{\prime}=\theta\left(I_{y^{\prime} y^{\prime}}-I_{x^{\prime} x^{\prime}}\right) \sin 2 \phi(z), \\
& I_{y y}^{\prime}=\theta\left(I_{x^{\prime} x^{\prime}}-I_{y^{\prime} y^{\prime}}\right) \sin 2 \phi(z), \\
& I_{x y}^{\prime}=\theta\left(I_{y^{\prime} y^{\prime}}-I_{x^{\prime} x^{\prime}}\right) \cos 2 \phi(z) .
\end{aligned}
$$


It is convenient to express Eq. (8) in the matrix form:

$$
\mathbf{d}=\mathbf{B a} \text { or in open form }\left\{\begin{array}{c}
c_{0} \\
c_{1} \\
c_{2} \\
d_{0} \\
d_{1} \\
d_{2}
\end{array}\right\}=\left[\begin{array}{cccccccc}
0 & 0 & \beta_{1} & \beta_{2} & 0 & 1 & \beta_{3} & \beta_{4} \\
0 & 0 & 0 & \beta_{5} & 0 & 0 & 2 & \beta_{6} \\
0 & 0 & 0 & 0 & 0 & 0 & 0 & 3 \\
0 & 1 & \beta_{7} & \beta_{8} & 0 & 0 & \beta_{1} & \beta_{2} \\
0 & 0 & 2 & \beta_{9} & 0 & 0 & 0 & \beta_{5} \\
0 & 0 & 0 & 3 & 0 & 0 & 0 & 0
\end{array}\right]\left\{\begin{array}{l}
a_{0} \\
a_{1} \\
a_{2} \\
a_{3} \\
b_{0} \\
b_{1} \\
b_{2} \\
b_{3}
\end{array}\right\},
$$

where $\mathbf{a}$ and $\mathbf{d}$ are named as independent and dependent coefficients vectors, respectively.

A similar procedure was applied to untwisted Timoshenko beam by Narayanaswami and Adelman [14], Dawe [15] and Petyt [16].

The new Timoshenko beam finite element has two nodes and four degrees of freedom per node, namely, two transverse deflections and two rotations (Fig. 2). The element displacement vector can be written as

$$
\mathbf{q}_{e}=\left\{\begin{array}{llllllll}
u_{1} & v_{1} & \theta_{x 1} & \theta_{y 1} & u_{2} & v_{2} & \theta_{x 2} & \theta_{y 2}
\end{array}\right\}^{\mathrm{T}} .
$$

Then, by using Eqs. (2)-(5) and (8), $\mathbf{q}_{e}$ can be expressed in terms of the independent coefficients vector as follows:

$$
\left.\begin{array}{c}
\mathbf{q}_{e}=\mathbf{C a} \\
\theta_{1} \\
\theta_{x 1} \\
u_{2} \\
v_{2} \\
\theta_{x 2} \\
\theta_{y 2}
\end{array}\right\}=\left[\begin{array}{cccccccc}
1 & 0 & 0 & 0 & 0 & 0 & 0 & 0 \\
0 & 0 & 0 & 0 & 1 & 0 & 0 & 0 \\
0 & 0 & \beta_{1} & \beta_{2} & 0 & 1 & \beta_{3} & \beta_{4} \\
0 & 1 & \beta_{7} & \beta_{8} & 0 & 0 & \beta_{1} & \beta_{2} \\
1 & L & L^{2} & L^{3} & 0 & 0 & 0 & 0 \\
0 & 0 & 0 & 0 & 1 & L & L^{2} & L^{3} \\
0 & 0 & \beta_{1} & \beta_{2}+\beta_{5} L & 0 & 1 & \beta_{3}+2 L & \beta_{4}+\beta_{6} L+3 L^{2} \\
0 & 1 & \beta_{7}+2 L & \beta_{8}+\beta_{9} L+3 L^{2} & 0 & 0 & \beta_{1} & \beta_{2}+\beta_{5} L
\end{array}\right]\left\{\begin{array}{l}
a_{0} \\
a_{1} \\
a_{2} \\
a_{3} \\
b_{0} \\
b_{1} \\
b_{2} \\
b_{3}
\end{array}\right\} .
$$

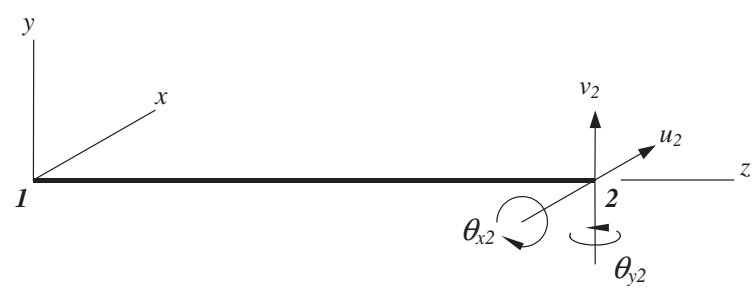

Fig. 2. Freedoms at the second node of the finite element. 
The linear and angular displacement functions can be written by using the independent and dependent coefficients vectors, respectively, as follows:

$$
\begin{gathered}
u(z)=\mathbf{P}_{u} \mathbf{a}=\left[\begin{array}{llllllll}
1 & z & z^{2} & z^{3} & 0 & 0 & 0 & 0
\end{array}\right] \mathbf{a}, \\
v(z)=\mathbf{P}_{v} \mathbf{a}=\left[\begin{array}{llllllll}
0 & 0 & 0 & 0 & 1 & z & z^{2} & z^{3}
\end{array}\right] \mathbf{a}, \\
\theta_{x}(z)=\mathbf{P}_{\theta_{x}} \mathbf{d}=\left[\begin{array}{llllll}
1 & z & z^{2} & 0 & 0 & 0
\end{array}\right] \mathbf{d}, \\
\theta_{y}(z)=\mathbf{P}_{\theta_{y}} \mathbf{d}=\left[\begin{array}{lllllll}
0 & 0 & 0 & 1 & z & z^{2}
\end{array}\right] \mathbf{d} .
\end{gathered}
$$

Now, Eqs. (16) and (17) can be expressed by using Eq. (11) as follows:

$$
\begin{aligned}
& \theta_{x}(z)=\mathbf{P}_{\theta_{x}} \mathbf{B a}, \\
& \theta_{y}(z)=\mathbf{P}_{\theta_{y}} \mathbf{B a} .
\end{aligned}
$$

The elastic potential energy of the finite element in Fig. 2 is written as [5]

$$
U=0.5 \int_{0}^{L}\left\{E\left(I_{x x} \theta_{x}^{\prime 2}+2 I_{x y} \theta_{x}^{\prime} \theta_{y}^{\prime}+I_{y y} \theta_{y}^{\prime 2}\right)+k A G\left(\left(u^{\prime}-\theta_{y}\right)^{2}+\left(v^{\prime}-\theta_{x}\right)^{2}\right)\right\} \mathrm{d} z,
$$

where primes denote differentiation with respect to $z$. Substituting Eqs. (7), (13), (14), (15), (18) and (19) into Eq. (20) gives

$$
U=0.5 \mathbf{q}_{e}^{\mathrm{T}} \mathbf{K}_{e} \mathbf{q}_{e},
$$

where $\mathbf{K}_{e}$ is the element stiffness matrix given by

$$
\mathbf{K}_{e}=\int_{0}^{L} \mathbf{C}^{-\mathrm{T}} \mathbf{K} \mathbf{C}^{-1} \mathrm{~d} z .
$$

$\mathbf{K}$ in Eq. (22) is given in Appendix B.

The kinetic energy of the pre-twisted thick beam is given as follows [5]:

$$
T=0.5 \int_{0}^{L} \rho\left\{A\left(\dot{u}^{2}+\dot{v}^{2}\right)+\left(I_{x x} \dot{\theta}_{x}^{2}+2 I_{x y} \dot{\theta}_{x} \dot{\theta}_{y}+I_{y y} \dot{\theta}_{y}^{2}\right)\right\} \mathrm{d} z,
$$

where the use of the overdot is a compact notation for differentiation with respect to time. Substituting Eqs. (7), (13), (14), (15),(18) and (19) into Eq. (23) gives

$$
T=0.5 \dot{\mathbf{q}}_{e}^{\mathrm{T}} \mathbf{M}_{e} \dot{\mathbf{q}}_{e},
$$

where $\mathbf{M}_{e}$ is the element mass matrix given by

$$
\mathbf{M}_{e}=\int_{0}^{L} \mathbf{C}^{-\mathrm{T}} \mathbf{M} \mathbf{C}^{-1} \mathrm{~d} z .
$$

$\mathbf{M}$ in Eq. (25) is given in Appendix B.

In order to compute $\mathbf{K}_{e}$ and $\mathbf{M}_{e}$ in the Eqs. (22) and (25), Gauss-Legendre 4-point numerical integration $[16,19]$ is used. 


\section{Finite element vibration analysis}

Element stiffness and mass matrices are obtained by using Gauss-Legendre numerical integration. The global mass and stiffness matrices are obtained by assembling these element matrices and applying the boundary conditions to them. By using the well-known procedures of vibration analysis, the eigenvalue problem can be given as [16]

$$
\left(\mathbf{K}_{g}-\Omega^{2} \mathbf{M}_{g}\right) \mathbf{q}_{g}=\mathbf{0},
$$

where $\mathbf{K}_{g}$ and $\mathbf{M}_{g}$ are global stiffness and mass matrices, respectively, and $\mathbf{q}_{g}$ is the global displacement vector, and $\Omega$ is the natural circular frequency. Developing a computer program in any language, such as APL or Matlab, the eigenvalue problem given in Eq. (26) is solved.

\section{Numerical results and discussion}

In order to validate the proposed finite element model for the vibration analysis of a pre-twisted Timoshenko beam, various numerical results are obtained and compared with available solutions in the published literature.

The first example to be considered is the case of lateral vibrations of an untwisted rectangular cross-section beam with both ends simply supported. In Table 1, comparison of the analytical results obtained from closed-form solution derived by Anderson [1], finite element solution with 20 and 40 elements given by Chen and Keer [9] and the present model with 10 elements is made. Excellent agreement is observed. The physical properties of the beam are given in Table 1 .

The second example is concerned with a cantilever pre-twisted beam treated experimentally by Carnegie [2] and by theoretical means by Lin et al. [11] and Subrahmanyam et al. [7]. The properties of the beam are given in Table 2. Although it is well known that shear coefficient depends on the geometry of the cross-section, it is taken the same as in the related references $[2,7,11]$ for comparison purposes. To show efficiency and convergence of the proposed model, the first four frequencies of the second example are calculated. For comparison, the present

Table 1

Comparison of coupled bending-bending frequencies of an untwisted, simple supported rectangular cross-section beam $(\mathrm{Hz})$

\begin{tabular}{|c|c|c|c|c|c|}
\hline \multirow[t]{2}{*}{ Mode } & \multirow{2}{*}{$\begin{array}{l}\text { Anderson } \\
\text { analytical [1] }\end{array}$} & \multicolumn{2}{|c|}{ Chen and Keer FEM [9] } & \multirow{2}{*}{$\begin{array}{l}\text { Present FEM } \\
10 \text { elements }\end{array}$} & \multirow{2}{*}{$\begin{array}{l}\text { Difference between } \\
\text { analytical and } \\
\text { present }(\%)\end{array}$} \\
\hline & & 20 elements & 40 elements & & \\
\hline 1 & 114.78 & 115.14 & 114.87 & 113.99 & 0.69 \\
\hline 2 & 333.46 & 334.44 & 333.70 & 331.21 & 0.67 \\
\hline 3 & 453.49 & 459.01 & 454.86 & 450.55 & 0.65 \\
\hline 4 & 1000.38 & 1027.41 & 1007.03 & 995.81 & 0.46 \\
\hline 5 & 1216.72 & 1229.63 & 1219.92 & 1211.76 & 0.41 \\
\hline
\end{tabular}

Data: length of beam $=101.6 \mathrm{~cm}$, width $=5.08 \mathrm{~cm}$, thickness $=15.24 \mathrm{~cm}$, shear coefficient $=5 / 6, E=206.8 \mathrm{GPa}$, $G=79.3 \mathrm{GPa}$, mass density $=7860 \mathrm{~kg} / \mathrm{m}^{3}$. 
Table 2

Convergence pattern and comparison of the frequencies of a cantilever pre-twisted uniform Timoshenko beam (Hz)

\begin{tabular}{lllll}
\hline Number of element & \multicolumn{2}{l}{ Mode number } & & \\
\cline { 2 - 5 } & 1 & 2 & 3 & 1087.7 \\
\hline 2 & 64.3 & 465.5 & 1041.7 & 1921.6 \\
4 & 62.3 & 327.3 & 986.3 & 1226.2 \\
6 & 62.0 & 313.6 & 965.4 & 1179.2 \\
8 & 61.9 & 309.3 & 956.1 & 1178.3 \\
10 & 61.8 & 307.3 & 951.2 & 1181.9 \\
12 & 61.8 & 306.3 & 948.3 & 1185.4 \\
14 & 61.8 & 305.6 & 946.6 & 1188.1 \\
16 & 61.8 & 305.3 & 945.4 & 1190.2 \\
18 & 61.8 & 305.0 & 944.5 & 1191.7 \\
20 & 61.8 & 304.8 & & 1193.0 \\
Lin et al. [11] & & & 917.0 & 1175.1 \\
Subrahmanyam et al. [7] & 61.7 & 300.9 & 955.1 & 1214.7 \\
Subrahmanyam et al. [7] & 62.0 & 305.1 & 937.0 & 1205.1 \\
Carnegie [2] & 61.9 & 304.7 & 920.0 & 1110.0 \\
\hline
\end{tabular}

Data: length of beam $=15.24 \mathrm{~cm}$, breadth $=2.54 \mathrm{~cm}$, depth $=0.17272 \mathrm{~cm}$, shear coefficient $=0.847458, E=206.85 \mathrm{GPa}$, $G=82.74 \mathrm{GPa}$, mass density $=7857.6 \mathrm{~kg} / \mathrm{m}^{3}$, pre-twist angle $=45^{\circ}$.

results as well as those given by other investigators are tabulated in Table 2. It is observed that the agreement between the present results and results of the other investigators is very good. The natural frequencies calculated by the proposed model converge very rapidly. Even when the number of the element is only 10, the present fundamental frequency is converged.

The last example is considered to evaluate the present finite element formulation for the effects of related parameters (e.g. twist angle, length, breadth-to-depth ratio) on the natural frequencies of the pre-twisted cantilever Timoshenko beams treated experimentally by Dawson et al. [4]. The natural frequencies are prescribed in terms of the frequency ratio $=\Omega / \Omega_{0}$, where $\Omega$ is the natural frequency of pre-twisted beam and $\Omega_{0}$ is the fundamental natural frequency of untwisted beam. The natural frequency ratios for the first five modes of vibration are obtained for two groups of cantilever beams of uniform rectangular cross-section by using 10 elements. First group includes the sets of beams of breadth $0.0254 \mathrm{~m}$ and length $0.3048 \mathrm{~m}$ and various breadth-to-depth ratios and pre-twist angle in the range $0-90^{\circ}$. The results for first group are shown in Figs. 3-5. Second group contains the sets of beams of breadth $0.0254 \mathrm{~m}$ and breadth-to-depth ratio $8 / 1$ and length ranging from 0.0762 to $0.508 \mathrm{~m}$ and pre-twist angle in the range $0-90^{\circ}$. The results for second group are shown in Fig. 3 and Figs. 6-8. It can clearly be seen in Figs. 3-8 that the agreement between present and experimental results is excellent. The natural frequency ratios obtained by employing 25 finite elements by Chen and Keer [9] and by using the differential transform by Chen and Ho [10] are also included for reference purposes in Fig. 3. It may also be inferred from Fig. 3 that present finite element formulation shows an improvement in finite element modelling. 


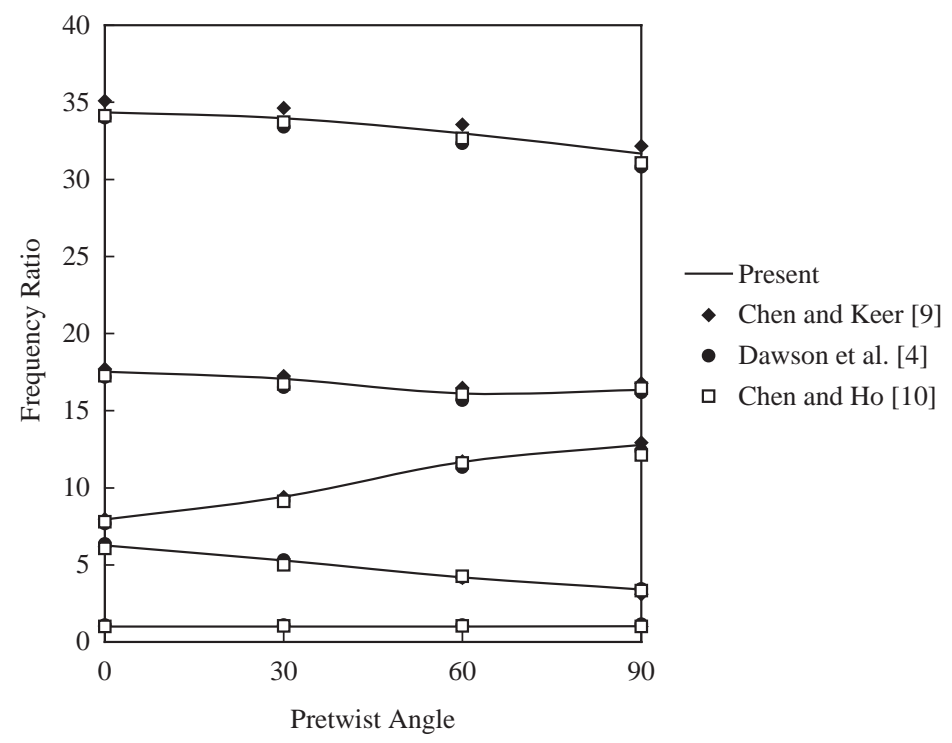

Fig. 3. Frequency ratio vs. twist angle: length $30.48 \mathrm{~cm}$, breadth $2.54 \mathrm{~cm}, b / h=8 / 1$.

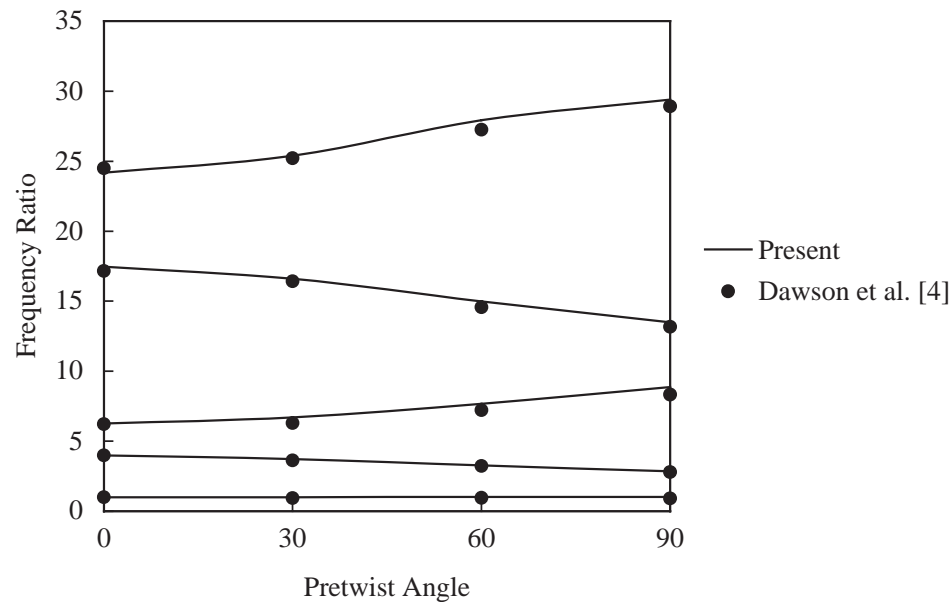

Fig. 4. Frequency ratio vs. twist angle: length $30.48 \mathrm{~cm}$, breadth $2.54 \mathrm{~cm}, b / h=4 / 1$.

\section{Conclusions}

The new eight-degree-of-freedom pre-twisted Timoshenko beam element is developed. The finite element model developed is based on two displacement fields that couple the transverse and angular displacements in two planes by satisfying the coupled differential equations of static equilibrium. The rotary inertia terms are included in "lumped" form at the nodes. The model has the considerable advantage of using few variables as nodal freedoms. Also, nodal variables 


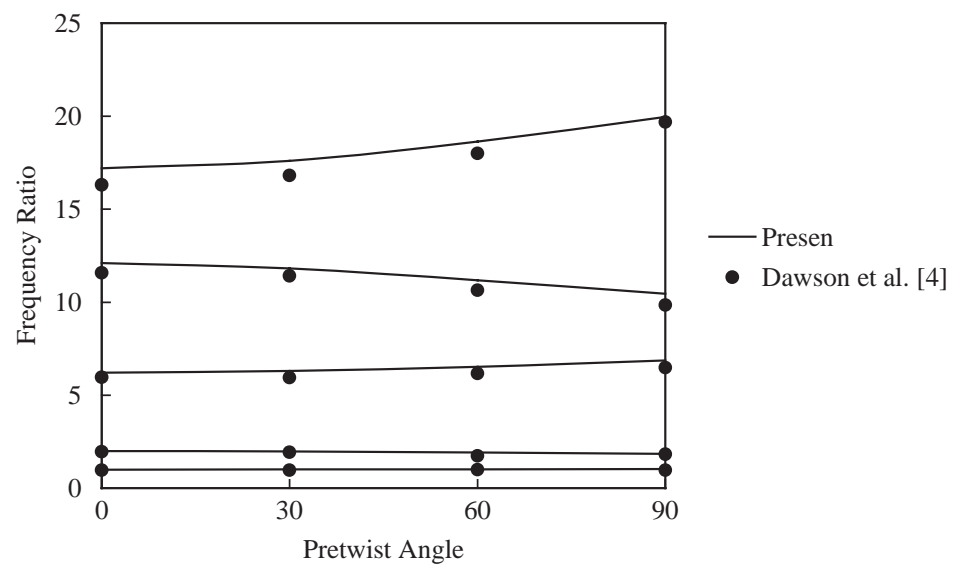

Fig. 5. Frequency ratio vs. twist angle: length $30.48 \mathrm{~cm}$, breadth $2.54 \mathrm{~cm}, b / h=2 / 1$.

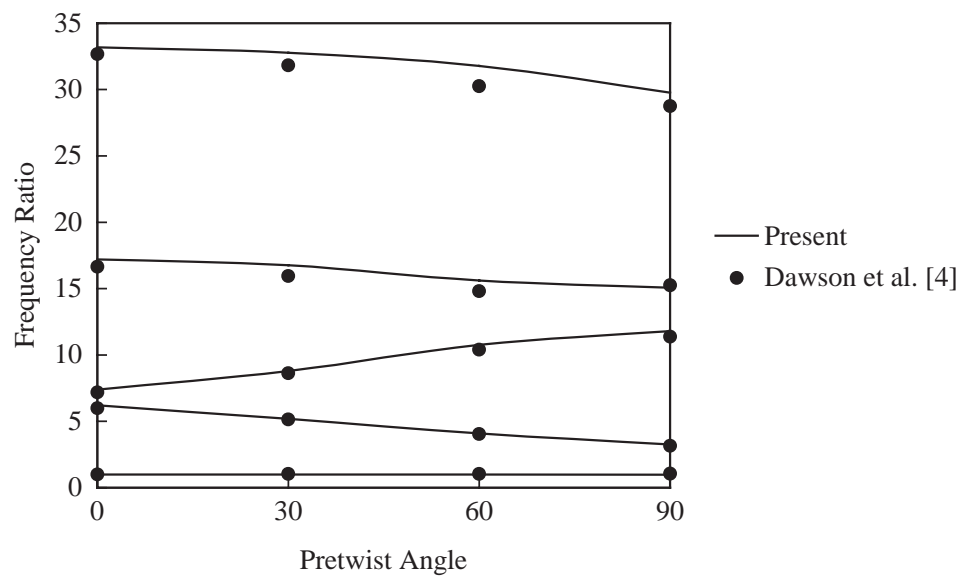

Fig. 6. Frequency ratio vs. twist angle: length $7.62 \mathrm{~cm}$, breadth $2.54 \mathrm{~cm}, b / h=8 / 1$.

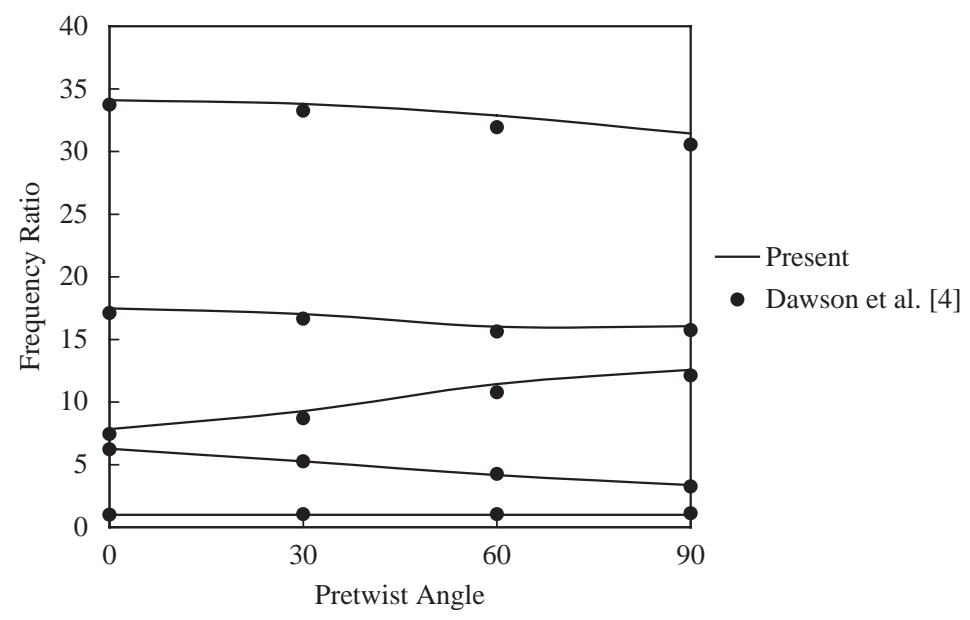

Fig. 7. Frequency ratio vs. twist angle: length $15.24 \mathrm{~cm}$, breadth $2.54 \mathrm{~cm}, b / h=8 / 1$. 


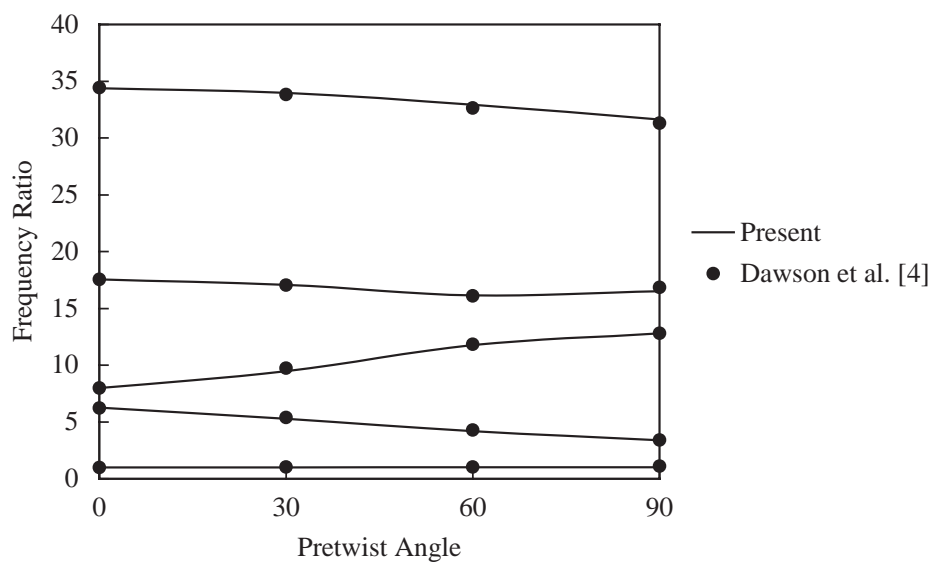

Fig. 8. Frequency ratio vs. twist angle; length $50.8 \mathrm{~cm}$, breadth $2.54 \mathrm{~cm}, b / h=8 / 1$.

facilitate the use of this element in the analysis of general structure involving other types of finite element having the same nodal freedoms.

The present model is verified for various parameters (such as twist angle, length, breadth-todepth ratio) in different range on the vibrations of the twisted beam treated experimentally by Carnegie [2] and Dawson et al. [4] and theoretically by other investigators [1,7,9-11] even with 10 elements.

\section{Appendix A. Differential equations of the motion of a pre-twisted Timoshenko beam for coupled bending-bending vibrations}

The differential equations of motion of a pre-twisted beam for coupled bending-bending vibrations are given as follows [3-5]:

$$
\begin{gathered}
\frac{\mathrm{d}}{\mathrm{d} z}\left(M_{x}\right)+V_{y}=-I_{x x} \rho \ddot{\theta}_{x}+I_{x y} \rho \ddot{\theta}_{y}, \\
\frac{\mathrm{d}}{\mathrm{d} z}\left(M_{y}\right)+V_{x}=I_{y y} \rho \ddot{\theta}_{y}-I_{x y} \rho \ddot{\theta}_{x}, \\
\frac{\mathrm{d}}{\mathrm{d} z}\left(V_{x}\right)=\rho A \ddot{u}, \\
\frac{\mathrm{d}}{\mathrm{d} z}\left(V_{y}\right)=\rho A \ddot{v}
\end{gathered}
$$

where

$$
\begin{gathered}
M_{x}=-E I_{x x} \theta_{x}^{\prime}-E I_{x y} \theta_{y}^{\prime}, \\
M_{y}=E I_{y y} \theta_{y}^{\prime}+E I_{x y} \theta_{x}^{\prime},
\end{gathered}
$$




$$
\begin{gathered}
V_{x}=k A G\left(u^{\prime}-\theta_{y}\right), \\
V_{y}=-k A G\left(v^{\prime}-\theta_{x}\right) .
\end{gathered}
$$

In Eqs. (A.1)-(A.8), dots and primes denote differentiation with respect to time and $z$, respectively. It should be noted that the formulae above are defined in the co-ordinate system shown in Fig. 1, which is different to the Refs. [3-5].

\section{Appendix B. K and M}

For the interested readers $\mathbf{K}$ and $\mathbf{M}$ are given below in open form:

$$
\begin{aligned}
\mathbf{K}= & E \mathbf{B}^{\mathrm{T}}\left\{I_{x x}(z) \mathbf{P}_{\theta_{x}}^{\prime \mathrm{T}} \mathbf{P}_{\theta_{x}}^{\prime}+I_{y y}(z) \mathbf{P}_{\theta_{y}}^{\prime \mathrm{T}} \mathbf{P}_{\theta_{y}}^{\prime}+I_{x y}(z)\left(\mathbf{P}_{\theta_{x}}^{\prime \mathrm{T}} \mathbf{P}_{\theta_{y}}^{\prime}+\mathbf{P}_{\theta_{y}}^{\prime \mathrm{T}} \mathbf{P}_{\theta_{x}}^{\prime}\right)\right\} \mathbf{B} \\
& +k A G\left\{\mathbf{P}_{u}^{\prime \mathrm{T}} \mathbf{P}_{u}^{\prime}+\mathbf{P}_{v}^{\prime \mathrm{T}} \mathbf{P}_{v}^{\prime}+\mathbf{B}^{\mathrm{T}}\left(\mathbf{P}_{\theta_{x}}^{\mathrm{T}} \mathbf{P}_{\theta_{x}}+\mathbf{P}_{\theta_{y}}^{\mathrm{T}} \mathbf{P}_{\theta_{y}}\right) \mathbf{B}\right\} \\
& -k A G\left\{\mathbf{B}^{\mathrm{T}}\left(\mathbf{P}_{\theta_{y}}^{\mathrm{T}} \mathbf{P}_{u}^{\prime}+\mathbf{P}_{\theta_{x}}^{\mathrm{T}} \mathbf{P}_{v}^{\prime}\right)+\left(\mathbf{P}_{u}^{\prime \mathrm{T}} \mathbf{P}_{\theta_{y}}+\mathbf{P}_{v}^{/ \mathrm{T}} \mathbf{P}_{\theta_{x}}\right) \mathbf{B}\right\}, \\
\mathbf{M}= & \rho A\left(\dot{\mathbf{P}}_{u}^{\mathrm{T}} \dot{\mathbf{P}}_{u}+\dot{\mathbf{P}}_{v}^{\mathrm{T}} \dot{\mathbf{P}}_{v}\right) \\
& +\rho\left\{\mathbf{B}\left(I_{x x}(z) \dot{\mathbf{P}}_{\theta_{x}}^{\mathrm{T}} \dot{\mathbf{P}}_{\theta_{x}}+I_{x y}(\mathbf{z})\left(\dot{\mathbf{P}}_{\theta_{x}}^{\mathrm{T}} \dot{\mathbf{P}}_{\theta_{y}}+\dot{\mathbf{P}}_{\theta_{y}}^{\mathrm{T}} \dot{\mathbf{P}}_{\theta_{x}}\right)+I_{y y}(z) \dot{\mathbf{P}}_{\theta_{y}}^{\mathrm{T}} \dot{\mathbf{P}}_{\theta_{y}}\right) \mathbf{B}\right\}
\end{aligned}
$$

\section{Appendix C. Nomenclature}

a independent coefficient vector

$a_{0}, a_{1}, a_{2}, a_{3}$ polynomial coefficients of the linear displacement in $x z$ plane

$A \quad$ cross-sectional area of the beam

$b_{0}, b_{1}, b_{2}, b_{3}$ polynomial coefficients of the linear displacement in $y z$ plane

$b \quad$ breadth of the beam

$c_{0}, c_{1}, c_{2}$ polynomial coefficients of the angular displacement about the $x$-axis

d dependent coefficient vector

$d_{0}, d_{1}, d_{2} \quad$ polynomial coefficients of the angular displacement about the $y$-axis

E modulus of elasticity

$G \quad$ modulus of rigidity

$h \quad$ depth of the beam

$I_{x x}, I_{y y} \quad$ area moments of inertia of the cross-section about $x x$ - and $y y$-axis

$I_{x y} \quad$ product moment of inertia of the cross-section about $x x-y y$ axes

$I_{x^{\prime} x^{\prime}}, I_{y^{\prime} y^{\prime}} \quad$ area moments of inertia of the cross-section about $x^{\prime} x^{\prime}$ - and $y^{\prime} y^{\prime}$-axis

$k \quad$ shear coefficient

$\mathbf{K}_{e} \quad$ element stiffness matrix

$L \quad$ length of the beam

$M_{x}, M_{y} \quad$ bending moments about $x$ - and $y$-axis

$\mathbf{M}_{e} \quad$ element mass matrix

$\mathbf{q}_{e} \quad$ element displacement vector 


$\begin{array}{ll}T & \text { kinetic energy } \\ u & \text { linear displacement in } x z \text { plane } \\ U & \text { strain energy } \\ v & \text { linear displacement in } y z \text { plane } \\ V_{x}, V_{y} & \text { shear forces in } x \text { and } y \text { direction } \\ x, y & \text { principal axes through the centroid at root section } \\ x^{\prime}, y^{\prime} & \text { principal axes through the centroid at any section } \\ z & \text { co-ordinate distance measured along beam } \\ \theta & \text { twist angle per unit length } \\ \theta_{x}, \theta_{y} & \text { angular displacement about the } x \text { - and } y \text {-axis } \\ \rho & \text { density } \\ \phi_{0} & \text { initial pre-twist angle of the finite element } \\ \phi & \text { pre-twist angle of the finite element at any section } \\ \Omega_{0} & \text { fundamental natural circular frequency of a untwisted beam } \\ \Omega & \text { natural circular frequency of a pre-twisted beam }\end{array}$

\section{References}

[1] R.A. Anderson, Flexural vibrations in uniform beams according to the Timoshenko theory, Journal of Applied Mechanics 20 (1953) 504-510.

[2] W. Carnegie, Vibration of pre-twisted cantilever blading, Proceedings of the Institution of Mechanical Engineers 173 (1959) 343-374.

[3] W. Carnegie, Vibration of pre-twisted cantilever blading allowing for rotary inertia and shear deflection, Journal of Mechanical Engineering Science 6 (1964) 105-109.

[4] B. Dawson, N.G. Ghosh, W. Carnegie, Effect of slenderness ratio on the natural frequencies of pre-twisted cantilever beams of uniform rectangular cross-section, Journal of Mechanical Engineering Science 13 (1971) $51-59$

[5] W. Carnegie, J. Thomas, The effects of shear deformation and rotary inertia on the lateral frequencies of cantilever beams in bending, Journal of Engineering for Industry Transactions of the American Society of Mechanical Engineers 94 (1972) 267-278.

[6] R.S. Gupta, S.S. Rao, Finite element eigenvalue analysis of tapered and twisted Timoshenko beams, Journal of Sound and Vibration 56 (1978) 187-200.

[7] K.B. Subrahmanyam, S.V. Kulkarni, J.S. Rao, Coupled bending-bending vibration of pre-twisted cantilever blading allowing for shear deflection and rotary inertia by the Reissner method, International Journal of Mechanical Sciences 23 (1981) 517-530.

[8] A. Rosen, Structural and dynamic behavior of pre-twisted rods and beams, Applied Mechanics Reviews 44 (1991) $483-515$.

[9] W.R. Chen, L.M. Keer, Transverse vibrations of a rotating twisted Timoshenko beam under axial loading, Journal of Vibrations and Acoustics 115 (1993) 285-294.

[10] C.K. Chen, S.H. Ho, Transverse vibration of a rotating twisted Timoshenko beams under axial loading using differential transform, International Journal of Mechanical Science 41 (1999) 1339-1356.

[11] S.M. Lin, W.R. Wang, S.Y. Lee, The dynamic analysis of nonuniformly pre-twisted Timoshenko beams with elastic boundary conditions, International Journal of Mechanical Science 43 (2001) 2385-2405.

[12] J.R. Banerjee, Free vibration analysis of a twisted beam using the dynamic stiffness method, International Journal of Solids and Structures 38 (2001) 6703-6722.

[13] S.S. Rao, R.S. Gupta, Finite element vibration analysis of rotating Timoshenko beams, Journal of Sound and Vibration 242 (2001) 103-124. 
[14] R. Narayanaswami, H.M. Adelman, Inclusion of transverse shear deformation in finite element displacement formulations, American Institute of Aeronautics and Astronautics Journal 12 (1974) 1613-1614.

[15] D.J. Dawe, A finite element for the vibration analysis of Timoshenko beams, Journal of Sound and Vibration 60 (1978) 11-20.

[16] M. Petyt, Introduction to Finite Element Vibration Analysis, Cambridge University Press, Cambridge, 1990.

[17] J.S. Przemieniecki, Theory of Matrix Structural Analysis, McGraw-Hill, New York, 1968.

[18] J.N. Reddy, An Introduction to the Finite Element Method, 2nd Edition, McGraw-Hill, New York, 1993.

[19] K.J. Bathe, Finite Element Procedures, Prentice-Hall, Englewood Cliffs, NJ, 1996. 